Pure and Applied Mathematics Quarterly

Volume 7, Number 4

(Special Issue:

In memory of Eckart Viehweg)

$1209-1240,2011$

\title{
Finite Group Actions, Rational Fixed Points and Weak Néron Models
}

Hélène Esnault and Johannes Nicaise

À la mémoire d'Eckart Viehweg, en souvenir de la lumière dont il nous inondait

\begin{abstract}
If $G$ is a finite $\ell$-group acting on an affine space $\mathbb{A}_{K}^{n}$ over a finite field $K$ of cardinality prime to $\ell$, Serre [32] shows that there exists a rational fixed point. We generalize this to the case where $K$ is a henselian discretely valued field of characteristic zero with algebraically closed residue field and with residue characteristic different from $\ell$. We also treat the case where the residue field is finite of cardinality $q$ such that $\ell$ divides $q-1$. To this aim, we study group actions on weak Néron models.
\end{abstract}

Keywords: Weak Néron Models, Rational Fixed Points, Finite Group Actions, Motivic Serre Invariant.

\section{INTRODUCTION}

Let $\ell$ be a prime number, and let $G$ be a finite $\ell$-group acting on an affine space $\mathbb{A}_{K}^{n}$ over a field $K$ of characteristic different from $\ell$ (here we view $\mathbb{A}_{K}^{n}$ as a $K$-variety, not as the $K$-group $\left.\mathbb{G}_{a, K}^{n}\right)$. Serre observes in [32] that the closed subscheme $\left(\mathbb{A}_{K}^{n}\right)^{G}$ of fixed points is not empty; see also Białynicki-Birula [1]. By standard arguments, it is enough to show this if $K$ is a finite field. Over a finite

Received: Sept. 15, 2010; Revised: Feb. 9, 2011.

The first author was partially supported by the DFG Leibniz Preis, the SFB/TR45, the ERC Advanced Grant 226257. The second author was partially supported by the Fund for Scientific Research - Flanders (project G.0415.10). 
field, non-trivial orbits of the set of rational points have cardinality divisible by $\ell$, thus there must exist fixed points that are rational. Serre raises the question [32] whether rational fixed points always exist, that is, whether $\left(\mathbb{A}_{K}^{n}\right)^{G}(K)$ is empty or not.

The aim of this note is to discuss Serre's question for a field $K$ whose Galois group is close to $\widehat{\mathbb{Z}}$, the Galois group of a finite field. The main theorem (5.17) and its corollary (5.18) assert that if $K$ is a henselian discretely valued field of characteristic 0 , with algebraically closed residue field and residue characteristic different from $\ell$, then Serre's question has a positive answer. Furthermore, if $K$ is henselian with finite residue field of cardinality $q$ such that $\ell$ divides $q-1$, then Serre's question has a positive answer as well.

While Serre's counting argument sketched above over a finite field $K$ is very elementary, one can also argue cohomologically. Serre shows that, for any base field $K$, the Gysin map in compactly supported étale cohomology

$$
H_{c}^{*-2 c}\left(\left(\mathbb{A}_{K}^{n}\right)^{G} \times_{K} K^{a}, \mathbb{F}_{\ell}(-c)\right) \stackrel{\gamma_{K^{a}}}{\longrightarrow} H_{c}^{*}\left(\mathbb{A}_{K^{a}}^{n}, \mathbb{F}_{\ell}\right)
$$

is an isomorphism, where $K^{a}$ is an algebraic closure of $K$ and $c$ is the codimension of $\left(\mathbb{A}_{K}^{n}\right)^{G}$ in $\mathbb{A}_{K}^{n}$. Now assume that $K$ is finite, of cardinality $q$. Since the Gysin map is Galois-equivariant and $\mathbb{A}_{K}^{n}$ has trivial cohomology, it follows from the isomorphism (1.1) that the trace of the geometric Frobenius on the graded cohomology space

$$
\bigoplus_{i=0}^{2 n-2 c} H_{c}^{i}\left(\left(\mathbb{A}_{K}^{n}\right)^{G} \times_{K} K^{a}, \mathbb{F}_{\ell}\right)
$$

is equal to $q^{n-c}$. The Grothendieck-Lefschetz trace formula allows one to conclude the existence of a rational fixed point.

The second author has shown that a similar trace formula exists when the base field is a strictly henselian discretely valued field $K$ of equicharacteristic zero (there are partial results in arbitrary characteristic [25], but there the situation is much more subtle due to issues of wild ramification). He showed in [24] that, for any $K$-variety $X$, the trace of any monodromy operator (i.e., any topological generator of the absolute Galois group $\left.\operatorname{Gal}\left(K^{a} / K\right)\right)$ on the compactly supported $\ell$-adic cohomology of $X$ is equal to the rational volume $s(X)$ of $X$. The rational volume is a certain measure for the set of rational points of $X$ (see Definition 3.13). If $X$ admits a weak Néron model $\mathcal{X}$ over the valuation ring of $K$, then 
$s(X)$ equals the $\ell$-adic Euler characteristic of the special fiber of $\mathcal{X}$. The invariant $s(X)$ vanishes if $X(K)=0$, so that it can be used to detect the existence of a rational point. The rational volume is a specialization of Loeser and Sebag's motivic Serre invariant [22] and its generalization in [24]; see Section 3.

The existence of such a trace formula is surprising, because one cannot characterize rational points with intersection theory over $K^{a}$, since the action of the monodromy operator cannot be represented by an algebraic cycle. The proof of the trace formula uses resolution of singularities and explicit computations on nearby cycles. It is a challenging problem to find a more conceptual proof that does not use resolution of singularities.

When applied to Serre's question, the trace formula and the isomorphism (1.1) imply that the rational volume of $\left(\mathbb{A}_{K}^{n}\right)^{G}$ is equal to one, so that $\left(\mathbb{A}_{K}^{n}\right)^{G}(K)$ is non-empty. We also consider the case where $K$ has mixed characteristic. Here we cannot use the trace formula due to issues of wild ramification. Rather than using the whole information contained in the rational volume, we consider it only modulo $\ell$. Under the assumption that the residue field of $K$ has characteristic different from $\ell$, we show that $s\left(\mathbb{A}_{K}^{n}\right)$ and $s\left(\left(\mathbb{A}_{K}^{n}\right)^{G}\right)$ are congruent modulo $\ell$. Intuitively, this can still be considered as a manifestation of the isomorphism (1.1) on the level of the rational volume. Since $s\left(\mathbb{A}_{K}^{n}\right)=1$, we find that $\left(\mathbb{A}_{K}^{n}\right)^{G}(K)$ is non-empty.

The geometric part of the work consists in showing the compatibility of weak Néron models with group actions.

Acknowledegements: This work was started during a summer school at the Feza Gürsey Institute in Istanbul in June 2010. We thank the Turkish mathematicians for their hospitality. We thank Nguyêñ Duy Tân and JeanPierre Serre for careful reading and interesting questions. The second author is grateful to Raf Cluckers for inspiring discussions. We thank the referee for a friendly precise reading and for valuable suggestions. In particular, Lemma 4.2 is due to him. We thank Vladimir Popov for kindly supplying the reference [1].

\section{Preliminaries AND Notations}

2.1. Notations. We denote by $R$ a discrete valuation ring with quotient field $K$ and perfect residue field $k$. We denote by $p$ the characteristic exponent of $k$ 
(thus $p=1$ if $k$ has characteristic zero, and $p$ equals the characteristic of $k$ if it is non-zero) and by $\mathfrak{m}$ the maximal ideal of $R$. We fix a strict henselization $R^{s h}$ of $R$, and we denote by $K^{s h}$ its quotient field. The residue field $k^{s}$ of $R^{s h}$ is a separable closure of $k$. We denote by $v_{K}$ the discrete valuation on $K$. We choose a value $\varepsilon$ in $] 0,1\left[\right.$ and we define an absolute value $|\cdot|_{K}$ on $K$ by $|0|_{K}=0$ and

$$
|x|_{K}=\varepsilon^{v_{K}(x)}
$$

for $x \in K^{\times}$. If $k$ is finite, of cardinality $q$, we take $\varepsilon=q^{-1}$. The absolute value $|\cdot|_{K}$ extends canonically to $K^{s h}$.

For any $R$-scheme $\mathcal{X}$, we put

$$
\begin{gathered}
\mathcal{X}_{k}=\mathcal{X} \times_{R} k \\
\mathcal{X}_{K}=\mathcal{X} \times_{R} K .
\end{gathered}
$$

For every scheme $S$, we denote by $S_{\text {red }}$ the maximal reduced closed subscheme of $S$. An $S$-variety is a reduced separated $S$-scheme of finite presentation.

If $F$ is a field with separable closure $F^{s}, X$ is a separated $F$-scheme of finite type, and $\ell$ is a prime invertible in $F$, we define the Euler characteristic with proper supports of $X$ by

$$
\chi_{c}(X)=\sum_{i \geq 0}(-1)^{i} \operatorname{dim} H_{c}^{i}\left(X \times_{F} F^{s}, \mathbb{Q}_{\ell}\right) .
$$

This Euler characteristic is independent of $\ell$. If $F$ has characteristic zero, this follows from comparison with singular cohomology; if $F$ is finite, from the cohomological interpretation of the zeta function $\left(\chi_{c}(X)\right.$ is equal to minus the degree of the Hasse-Weil zeta function of $X$ ); the general case is deduced from the finite field case by a spreading out argument and proper base change.

For any set $\mathscr{S}$, we denote its cardinality by $|\mathscr{S}|$.

2.2. Group actions. Let $G$ be a finite group. We say that an action of $G$ on a scheme $S$ is good if every orbit is contained in an affine open subscheme of $S$. This condition is automatically fulfilled, for instance, if $S$ is quasi-projective over an affine scheme, by $[21,3.3 .36(\mathrm{~b})]$.

Let $S$ be a scheme, with trivial $G$-action. We denote by $(\mathrm{Sch} / S)$ the category of $S$-schemes, and by $(G$-Sch $/ S)$ the category of $S$-schemes with $G$-action. By 
[7, Propositon 3.1], the functor

$$
(\mathrm{Sch} / S) \rightarrow(G-\mathrm{Sch} / S)
$$

that endows an $S$-scheme with the trivial $G$-action has a right adjoint, which we denote by

$$
(\cdot)^{G}:(G-\mathrm{Sch} / S) \rightarrow(\mathrm{Sch} / S): X \mapsto X^{G} .
$$

The $S$-scheme $X^{G}$ is called the fixed locus of the $G$-action on $X$. By definition, it represents the functor

$$
(\mathrm{Sch} / S) \rightarrow(\mathrm{Sets}): T \mapsto X(T)^{G} .
$$

The functor $(\cdot)^{G}$ commutes with arbitrary base change $S^{\prime} \rightarrow S$. If $X$ is separated over $S$, then the tautological morphism

$$
X^{G} \rightarrow X
$$

is a closed immersion [7, loc.cit.].

Proposition 2.1 (also [7], Proposition 3.4). If $X \rightarrow S$ is smooth, and $|G|$ is invertible on $X$, then $X^{G} \rightarrow S$ is smooth.

Remark 2.2. Proposition 2.1 was shown in [16, Prop. 1.3] when $S$ is the spectrum of an algebraically closed field.

2.3. Grothendieck rings. Let $F$ be a field. We denote by $K_{0}\left(\operatorname{Var}_{F}\right)$ the Grothendieck ring of varieties over $F$. As an abelian group, $K_{0}\left(\operatorname{Var}_{F}\right)$ is defined by the following presentation:

- generators: isomorphism classes $[X]$ of separated $F$-schemes of finite type $X$,

- relations: if $X$ is a separated $F$-scheme of finite type and $Y$ is a closed subscheme of $X$, then

$$
[X]=[Y]+[X \backslash Y]
$$

These relations are called scissor relations.

By the scissor relations, one has $[X]=\left[X_{\text {red }}\right]$ for every separated $F$-scheme of finite type $X$, and $[\emptyset]=0$. We endow the group $K_{0}\left(\operatorname{Var}_{F}\right)$ with the unique ring structure such that

$$
[X] \cdot\left[X^{\prime}\right]=\left[X \times_{F} X^{\prime}\right]
$$


for all $F$-varieties $X$ and $X^{\prime}$. The identity element for the multiplication is the class $[\operatorname{Spec} F]$ of the point. For a detailed survey on the Grothendieck ring of varieties, we refer to [27].

We denote by

$$
K_{0}^{\bmod }\left(\operatorname{Var}_{F}\right)
$$

the modified Grothendieck ring of varieties over $F[27, \S 3.8]$. This is the quotient of $K_{0}\left(\operatorname{Var}_{F}\right)$ by the ideal $\mathcal{I}_{F}$ generated by elements of the form

$$
[X]-[Y]
$$

where $X$ and $Y$ are separated $F$-schemes of finite type such that there exists a finite, surjective, purely inseparable $F$-morphism

$$
Y \rightarrow X
$$

For instance, if $F$ has characteristic $p>0, X \rightarrow S$ is a morphism of $F$-varieties and $X^{(p)}$ is the pullback of $X$ over the absolute Frobenius morphism on $S$, then $\left[X^{(p)}\right]=[X]$ in $K_{0}^{\bmod }\left(\operatorname{Var}_{F}\right)$, since the relative Frobenius

$$
\text { Frob }_{X / S}: X \rightarrow X^{(p)}
$$

is a finite, surjective, purely inseparable $F$-morphism.

If $F$ has characteristic zero, then it is easily seen that $\mathcal{I}_{F}$ is the zero ideal $[27,3.11]$. It is not known if $\mathcal{I}_{F}$ is non-zero if $F$ has positive characteristic. In particular, if $F^{\prime}$ is a non-trivial finite purely inseparable extension of $F$, it is not known whether $\left[\operatorname{Spec} F^{\prime}\right] \neq 1$ in $K_{0}\left(\operatorname{Var}_{F}\right)$.

Let $G$ be a finite group. We denote by $K_{0}\left(G-\operatorname{Var}_{F}\right)$ the equivariant $G$ rothendieck ring of $F$-varieties with good $G$-action. It is the quotient of the free abelian group generated by isomorphism classes $[X]$ of separated $F$-schemes of finite type $X$ with good $G$-action, by the subgroup generated by elements of the form $[X]-[Y]-[X \backslash Y]$ with $X$ a separated $F$-scheme of finite type with good $G$-action and $Y$ a $G$-stable closed subscheme of $X$. The product on $K_{0}\left(G-\operatorname{Var}_{F}\right)$ is defined by

$$
[X] \cdot\left[X^{\prime}\right]=\left[X \times_{F} X^{\prime}\right]
$$

where $G$ acts diagonally on $X \times_{F} X^{\prime}$.

With a slight abuse of notation, we denote by the same symbol $\mathbb{L}_{F}$ the class of $\mathbb{A}_{F}^{1}$ - with trivial $G$-action, if applicable - in all of the above Grothendieck rings. 
If $R$ has equal characteristic, then we set

$$
K_{0}^{R}\left(\operatorname{Var}_{k}\right)=K_{0}\left(\operatorname{Var}_{k}\right) .
$$

If $R$ has mixed characteristic, then we set

$$
K_{0}^{R}\left(\operatorname{Var}_{k}\right)=K_{0}^{\bmod }\left(\operatorname{Var}_{k}\right)
$$

There exists a unique ring morphism

$$
\chi_{c}: K_{0}^{R}\left(\operatorname{Var}_{k}\right) /\left(\mathbb{L}_{k}-1\right) \rightarrow \mathbb{Z}
$$

that sends the class of a separated $k$-scheme of finite type $X$ to the Euler characteristic with proper supports $\chi_{c}(X)$ defined in section 2.1 (see [27, 4.2 and 4.14]). Moreover, if $k$ is finite, of cardinality $q$, there exists a unique ring morphism

$$
\sharp: K_{0}^{R}\left(\operatorname{Var}_{k}\right) /\left(\mathbb{L}_{k}-1\right) \rightarrow \mathbb{Z} /(q-1)
$$

that sends the class of a separated $k$-scheme of finite type $X$ to the residue class modulo $(q-1)$ of $|X(k)|$ (the cardinality of the set of $k$-rational points on $X$ ) [27, loc.cit.].

\section{THE SERRE INVARIANT}

Definition 3.1. We say that a $K$-variety $X$ is bounded if the set $X\left(K^{s h}\right)$ is bounded in $X$ in the sense of $[3,1.1 .2]$.

Every proper $K$-variety is bounded, by $[3,1.1 .6]$. If $n$ is a strictly positive integer, then a closed subvariety $X$ of

$$
\mathbb{A}_{K}^{n}=\operatorname{Spec} K\left[x_{1}, \ldots, x_{n}\right]
$$

is bounded if and only if, for every $i \in\{1, \ldots, n\}$, the function

$$
X\left(K^{s h}\right) \rightarrow \mathbb{R}^{+}: a \mapsto\left|x_{i}(a)\right|_{K}
$$

is bounded. In particular, $\mathbb{A}_{K}^{n}$ is not bounded.

Proposition 3.2 ([3], Corollary 3.5.7). A K-variety $X$ is bounded if and only if there exists an $R$-variety $\mathcal{X}$ endowed with a $K$-isomorphism

$$
\mathcal{X}_{K} \rightarrow X
$$


such that the natural map

$$
\mathcal{X}\left(R^{s h}\right) \rightarrow X\left(K^{s h}\right)
$$

is a bijection.

Definition 3.3. Let $X$ be a smooth $K$-variety. $A$ weak Néron model for $X$ is a smooth $R$-variety $\mathcal{X}$ endowed with an isomorphism

$$
\mathcal{X}_{K} \rightarrow X
$$

such that the natural map

$$
\mathcal{X}\left(R^{s h}\right) \rightarrow X\left(K^{s h}\right)
$$

is a bijection.

Note that $X\left(K^{s h}\right)$ is empty if and only if the special fiber $\mathcal{X}_{k}$ is empty, by [3, 2.3.5] (applied to $\left.\mathcal{X} \times_{R} R^{s h}\right)$. Thus if $X\left(K^{s h}\right)$ is empty, then up to isomorphism, $X$ is the unique weak Néron model of $X$.

Theorem 3.4. If $X$ is a smooth $K$-variety, then $X$ admits a weak Néron model if and only if $X$ is bounded.

Proof. If $X$ admits a weak Néron model, then $X$ is bounded by Proposition 3.2. Assume, conversely, that $X$ is bounded, and let $\mathcal{X}$ be an $R$-model of $X$ as in Proposition 3.2. By $[3,3.4 .2]$, there exists a projective morphism $\mathcal{X}^{\prime} \rightarrow \mathcal{X}$ such that the induced morphism $\mathcal{X}_{K}^{\prime} \rightarrow \mathcal{X}_{K}$ is an isomorphism and every element of $\mathcal{X}^{\prime}\left(R^{s h}\right)$ factors through the $R$-smooth locus $\operatorname{Sm}\left(\mathcal{X}^{\prime}\right)$ of $\mathcal{X}^{\prime}$. By the valuative criterion of properness, the map $\mathcal{X}^{\prime}\left(R^{s h}\right) \rightarrow \mathcal{X}\left(R^{s h}\right)$ is a bijection. It follows that $\operatorname{Sm}\left(\mathcal{X}^{\prime}\right)$ is a weak Néron model of $X$.

Definition 3.5. Let $X$ be a $K$-variety. We say that $X$ is of type $(N)$ if the $K$ smooth locus $\operatorname{Sm}(X)$ of $X$ contains all $K^{\text {sh }}$-points of $X$ and $\operatorname{Sm}(X)$ is bounded.

In particular, every smooth and proper $K$-variety is of type (N). If $X$ is of type $(\mathrm{N})$, then $\operatorname{Sm}(X)$ admits a weak Néron model.

Theorem 3.6. Let $X$ be a $K$-variety of type $(N)$, and let $\mathcal{X}$ be a weak Néron model for $\operatorname{Sm}(X)$. The class of $\mathcal{X}_{k}$ in the ring

$$
K_{0}^{R}\left(\operatorname{Var}_{k}\right) /\left(\mathbb{L}_{k}-1\right)
$$

only depends on $X$, and not on the choice of a weak Néron model $\mathcal{X}$. 
Proof. This result was originally proven by Loeser and Sebag for smooth quasicompact rigid $K$-varieties and their formal weak Néron models, under the assumption that $K$ is complete; see [22, 4.5.3]. It was extended to smooth and bounded rigid $K$-varieties in [26, 5.11]. A rigid $K$-variety $X$ is called bounded if it admits a quasi-compact open subset $U$ such that for every finite unramified extension $K^{\prime}$ of $K, U$ contains all $K^{\prime}$-points of $X$.

Theorem 3.6 is easily deduced from this case, as follows. Replacing $X$ by $\operatorname{Sm}(X)$, we may assume that $X$ is smooth. Denote by $\widehat{R}$ and $\widehat{K}$ the completions of $R$ and $K$, respectively. If $X$ is a smooth $K$-variety of type $(\mathrm{N})$, then the rigid analytification $\left(X \times_{K} \widehat{K}\right)^{\text {rig }}$ is smooth and bounded, by [6, 5.2.1] and [24, 4.3]. Moreover, if $\mathcal{X}$ is a weak Néron model for $X$, then its formal $\mathfrak{m}$-adic completion $\widehat{\mathcal{X}}$ over $\widehat{R}$ is a formal weak Néron model for $\left(X \times_{K} \widehat{K}\right)^{\text {rig }}$, by $[24,4.9]$, and the special fibers $\mathcal{X}_{k}$ and $\widehat{\mathcal{X}} \times{ }_{\operatorname{Spf}} \widehat{R}$ Spec $k$ are canonically isomorphic.

Definition 3.7. Let $X$ be a $K$-variety of type $(N)$, and let $\mathcal{X}$ be a weak Néron model for $\operatorname{Sm}(X)$. The motivic Serre invariant of $X$, denoted by $S(X)$, is the class of $\mathcal{X}_{k}$ in the ring

$$
K_{0}^{R}\left(\operatorname{Var}_{k}\right) /\left(\mathbb{L}_{k}-1\right)
$$

This definition only depends on $X$, by Theorem 3.6. Note that, if $X$ is a $K$ variety such that $X\left(K^{s h}\right)$ is empty, then $X$ is of type $(\mathrm{N})$ and $S(X)=0$. More generally, we can view $S(X)$ as a measure for the set of $K^{\text {sh }}$-rational points on $X$ : if $X$ has pure dimension $d$, then one can think of $X\left(K^{s h}\right)$ as a family of open unit balls in $\left(K^{s h}\right)^{d}$ parameterized by $\mathcal{X}_{k}$ via the specialization map

$$
s p: X\left(K^{s h}\right)=\mathcal{X}\left(R^{s h}\right) \rightarrow \mathcal{X}_{k}\left(k^{s}\right) .
$$

If $K$ is complete, this intuitive picture can be made more precise using the language of rigid geometry [29, 2.5].

Remark 3.8. In [22] and [24], the motivic Serre invariant was defined with values in

$$
K_{0}\left(\operatorname{Var}_{k}\right) /\left(\mathbb{L}_{k}-1\right) .
$$

In the meantime, Julien Sebag and the second author discovered a flaw in the change of variables formula for motivic integrals in mixed characteristic, upon which the proof of Theorem 3.6 was based. To correct it, one has to replace $K_{0}\left(\operatorname{Var}_{k}\right)$ by $K_{0}^{R}\left(\operatorname{Var}_{k}\right)$. This is explained in detail in [28] and [29]. The correction is harmless for all applications, since all realization morphisms of $K_{0}\left(\operatorname{Var}_{k}\right)$ that 
are used in practice factor through $K_{0}^{\bmod }\left(\operatorname{Var}_{k}\right)$ [27, 4.13]. In fact, if $k$ has positive characteristic, it is not even known if the projection

$$
K_{0}\left(\operatorname{Var}_{k}\right) \rightarrow K_{0}^{\bmod }\left(\operatorname{Var}_{k}\right)
$$

is an isomorphism.

The notion of motivic Serre invariant was first introduced by Loeser and Sebag in $[22, \S 4.5]$. It was inspired by Serre's invariant that classifies compact manifolds over a non-archimedean local field [31]. Assume that $K$ is complete and $k$ finite, of cardinality $q$. Let $M$ be a compact $K$-analytic manifold of pure dimension $d$ (in the naïve sense, not a rigid variety; see [5] and $[13, \S 2.4]$ ). Serre showed that $M$ is isomorphic to a disjoint union of closed unit balls $R^{d}$, and that the number $N$ of balls is well-defined modulo $(q-1)$. We call the class of $N$ in $\mathbb{Z} /(q-1)$ the Serre invariant of $M$, denoted by $S_{\sharp}(M)$.

Let $X$ be a $K$-variety of type (N). The space $X(K)$, with its $K$-adic topology, carries a canonical structure of compact $K$-analytic manifold, which we denote by $X^{\text {an }}$. The following Proposition compares the Serre invariant of $X^{\text {an }}$ to the motivic Serre invariant of $X$.

Proposition 3.9. Assume that $K$ is complete and $k$ finite, of cardinality $q$. Let $X$ be a $K$-variety of type $(N)$, of pure dimension $d$. Then the Serre invariant $S_{\sharp}\left(X^{\mathrm{an}}\right)$ of $X^{\text {an }}$ is the image of $S(X)$ under the point counting realization

$$
\sharp: K_{0}^{R}\left(\operatorname{Var}_{k}\right) /\left(\mathbb{L}_{k}-1\right) \rightarrow \mathbb{Z} /(q-1):[X] \mapsto|X(k)| .
$$

Proof. This was shown in $[22,4.6 .3]$ in the case where $X$ is a smooth quasicompact rigid $K$-variety. Proposition 3.9 is easily deduced from that case, as follows. We may assume that $X$ is smooth. Let $\mathcal{X}$ be a weak Néron model for $X$, and let $\widehat{\mathcal{X}}$ be its formal $\mathfrak{m}$-adic completion. The generic fiber $\widehat{\mathcal{X}}_{\eta}$ is a smooth quasi-compact open rigid subvariety of the rigid analytification $X^{\text {rig }}$ of $X$ that contains all $K$-points of $X^{\text {rig }}$, so the $K$-analytic manifold associated to $X$ is canonically isomorphic to the $K$-analytic manifold associated to $\widehat{\mathcal{X}}_{\eta}$. Moreover, the motivic Serre invariants of $\widehat{\mathcal{X}}_{\eta}$ and $X$ are the same: since $\widehat{\mathcal{X}}$ is a formal weak Néron model of $\widehat{\mathcal{X}}_{\eta}$, both motivic Serre invariants are equal to the class of $\mathcal{X}_{k}$ in $K_{0}^{R}\left(\operatorname{Var}_{k}\right) /(\mathbb{L}-1)$.

Let us briefly sketch the proof of [22, 4.6.3], translated to the set-up of Proposition 3.9. We may assume that $X$ is smooth. Let $\mathcal{X}$ be a weak Néron 
model for $X$. One has a specialization map

$$
s p_{\mathcal{X}}: X(K)=\mathcal{X}(R) \rightarrow \mathcal{X}(k)
$$

defined by reduction modulo the maximal ideal of $R$. For every point $x$ of $\mathcal{X}(k)$, the fiber $s p_{\mathcal{X}}^{-1}(x)$ is an open subset of $X(K)$, and it inherits from $X^{\text {an }}$ a structure of $K$-analytic manifold. Smoothness of $\mathcal{X}$ over $R$ implies that $s p_{\mathcal{X}}^{-1}(x)$ is isomorphic to the closed unit disc $R^{d}$. As a $K$-analytic manifold, $X^{\text {an }}$ is isomorphic to the disjoint union

$$
\bigsqcup_{x \in \mathcal{X}(k)} s p_{\mathcal{X}}^{-1}(x)
$$

so that the $p$-adic Serre invariant of $X^{\text {an }}$ equals the class of $|\mathcal{X}(k)|$ in $\mathbb{Z} /(q-1)$. This is precisely the image of

$$
S(X)=\left[\mathcal{X}_{k}\right]
$$

under the point counting realization

$$
\sharp: K_{0}^{R}\left(\operatorname{Var}_{k}\right) /\left(\mathbb{L}_{k}-1\right) \rightarrow \mathbb{Z} /(q-1) .
$$

Lemma 3.10. Let $X$ be a $K$-variety, and denote by $\widehat{K}$ the completion of $K$. Then $X$ is of type (N) if and only if $X \times_{K} \widehat{K}$ is of type (N). Moreover,

$$
S(X)=S\left(X \times_{K} \widehat{K}\right) .
$$

Proof. By the canonical isomorphism

$$
\operatorname{Sm}\left(X \times_{K} \widehat{K}\right) \cong \operatorname{Sm}(X) \times_{K} \widehat{K}
$$

we may assume that $X$ is smooth. Then $X$ is bounded if and only if $X \times_{K} \widehat{K}$ is bounded, by [24, 4.4]. Denote by $\widehat{R}$ the completion of $R$. If $\mathcal{X}$ is a weak Néron model of $X$, then $\mathcal{X} \times_{R} \widehat{R}$ is a weak Néron model of $X \times_{K} \widehat{K}$, by [3, 3.6.7], and the special fibers of these weak Néron models are canonically isomorphic.

If $K$ has characteristic zero, the second author extended the construction of the motivic Serre invariant to arbitrary $K$-varieties, in the following way.

Theorem 3.11. Assume that $K$ has characteristic zero. There exists a unique ring homomorphism

$$
S: K_{0}\left(\operatorname{Var}_{K}\right) \rightarrow K_{0}^{R}\left(\operatorname{Var}_{k}\right) /\left(\mathbb{L}_{k}-1\right)
$$


that sends $[Y]$ to $S(Y)$ for every smooth and proper $K$-variety $Y$. The morphism $S$ sends $\mathbb{L}_{K}$ to 1 .

Let $X$ be a $K$-variety and $U$ be a subvariety of $X$. If $U$ contains all the points in $X\left(K^{\text {sh }}\right), U$ is smooth over $K$ and $U$ admits a weak Néron model $\mathcal{U}$, then $S([X])$ is the class of $\mathcal{U}_{k}$ in $K_{0}^{R}\left(\operatorname{Var}_{k}\right) /\left(\mathbb{L}_{k}-1\right)$. In particular, if $X$ has type $(N)$, then $S([X])=S(X)$.

Proof. If $K$ is complete, this is $[24,5.4]$. The proof is based on Weak Factorization (it uses Bittner's presentation of the Grothendieck ring [2]) and on a generalization of Néron smoothening to pairs of $K$-varieties to control the behaviour of the motivic Serre invariant under blow-up.

The general case can easily be deduced as follows. We denote by $\widehat{K}$ the completion of $K$ and by

$$
S_{\widehat{K}}: K_{0}\left(\operatorname{Var}_{\widehat{K}}\right) \rightarrow K_{0}^{R}\left(\operatorname{Var}_{k}\right) /\left(\mathbb{L}_{k}-1\right)
$$

the ring homomorphism from $[24,5.4]$. Uniqueness of $S$ follows from the fact that $K_{0}\left(\operatorname{Var}_{K}\right)$ is generated by the classes of smooth and projective $K$-varieties, by the scissor relations and Hironaka's resolution of singularities. To prove existence, we define $S$ to be the composition of the base change morphism

$$
K_{0}\left(\operatorname{Var}_{K}\right) \rightarrow K_{0}\left(\operatorname{Var}_{\widehat{K}}\right):[X] \mapsto\left[X \times_{K} \widehat{K}\right]
$$

with

$$
S_{\widehat{K}}: K_{0}\left(\operatorname{Var}_{\widehat{K}}\right) \rightarrow K_{0}^{R}\left(\operatorname{Var}_{k}\right) /\left(\mathbb{L}_{k}-1\right) .
$$

It follows from Lemma 3.10 that $S([Y])=S(Y)$ for every $K$-variety $Y$ of type (N). In particular, if $Y\left(K^{s h}\right)$ is empty, then $S([Y])=0$. If $X$ and $U$ are as in the statement, then we can partition $X \backslash U$ into subvarieties of $X$ without $K^{s h}$-points. Additivity of $S$ implies that $S([X])=S([U])$, and we know that $S([U])=S(U)$ because $U$ is of type $(\mathrm{N})$. By definition, $S(U)$ is the class of $\mathcal{U}_{k}$ in $K_{0}^{R}\left(\operatorname{Var}_{k}\right) /\left(\mathbb{L}_{k}-1\right)$.

Definition 3.12 ([24], Definition 5.5). Assume that $K$ has characteristic zero. For any $K$-variety $X$, we define the motivic Serre invariant of $X$ by

$$
S(X)=S([X]) \quad \in K_{0}^{R}\left(\operatorname{Var}_{k}\right) /\left(\mathbb{L}_{k}-1\right) .
$$

If $X$ is of type (N), this definition is compatible with Definition 3.7, by Theorem 3.11 . 
Definition 3.13. Let $X$ be a $K$-variety. Assume either that $K$ has characteristic zero, or that $X$ is of type $(N)$. We define the rational volume of $X$, denoted by $s(X)$, as

$$
s(X)=\chi_{c}(S(X)) \quad \in \mathbb{Z} .
$$

Remark 3.14. If $X$ has type (N) and $\mathcal{X}$ is a weak Néron model of $\operatorname{Sm}(X)$, then by definition, $s(X)=\chi_{c}\left(\mathcal{X}_{k}\right)$. To our knowledge, there is no general proof of the independence of $s(X)$ of the choice of $\mathcal{X}$ that does not use the change of variables formula for motivic integrals. If the residue field $k$ has characteristic zero, this independence property can also be deduced from the trace formula in Theorem 6.1 .

Example 3.15. Assume that $K$ has characteristic zero. For every integer $n>0$, we have $S\left(\mathbb{A}_{K}^{n}\right)=1$ and $s\left(\mathbb{A}_{K}^{n}\right)=1$, because $\left[\mathbb{A}_{K}^{n}\right]=\mathbb{L}_{K}^{n}$ in $K_{0}\left(\operatorname{Var}_{K}\right)$ and the morphism $S$ in Theorem 3.11 is a ring morphism that sends $\mathbb{L}_{K}$ to 1 .

The rational volume $s(X)$ vanishes if $X\left(K^{s h}\right)$ is empty. So one way to detect the existence of a $K^{s h}$-rational point on $X$ is to show the non-vanishing of the rational volume of $X$. If $R$ is henselian and the residue field $k$ is finite, the following proposition provides a similar technique to detect the existence of a $K$-rational point.

Proposition 3.16. Suppose that $R$ is henselian and that $k$ is finite, of cardinality $q$. Let $X$ be a $K$-variety. Assume either that $K$ has characteristic zero, or that $X$ is of type $(N)$. If

$$
\sharp S(X) \neq 0
$$

in $\mathbb{Z} /(q-1)$, then $X(K)$ is non-empty.

Proof. If $X$ is of type $(\mathrm{N})$, then $\operatorname{Sm}(X)$ admits a weak Néron model $\mathcal{X}$ over $R$, and

$$
\sharp S(X)=\left|\mathcal{X}_{k}(k)\right| \bmod (q-1) .
$$

Since $R$ is henselian and $\mathcal{X}$ is smooth over $R$, any element of $\mathcal{X}_{k}(k)$ lifts to an element of $\mathcal{X}(R)=X(K)$, so that $X(K)$ is non-empty if $\sharp S(X) \neq 0$.

It remains to prove the case where $K$ has characteristic zero. Here we can proceed by induction on the dimension of $X$. If $X$ has dimension zero, then $X$ is of type $(\mathrm{N})$ and we are done. Assume that $\operatorname{dim}(X)>0$, and that the result holds for all $K$-varieties of strictly smaller dimension. Since $K$ has characteristic 
zero and $X$ is reduced, the $K$-smooth locus $\operatorname{Sm}(X)$ is open and dense in $X$, and $Y=X \backslash \operatorname{Sm}(X)$ has strictly smaller dimension than $X$. Since

$$
\sharp S(\operatorname{Sm}(X))+\sharp S(Y)=\sharp S(X) \neq 0,
$$

we know that $\sharp S(Y) \neq 0$ or $\sharp S(\operatorname{Sm}(X)) \neq 0$. If $\sharp S(Y) \neq 0$ then $Y$ has a $K$-rational point by the induction hypothesis, so that $X$ also has a $K$-rational point. Thus, we may assume that $\sharp S(\operatorname{Sm}(X)) \neq 0$, and it suffices to consider the case where $X$ is smooth over $K$. By additivity of $\sharp S(\cdot)$, we may also assume that $X$ is connected.

Let $\bar{X}$ be a smooth compactification of $X$, and put $Z=\bar{X} \backslash X$. Since

$$
\sharp S(\bar{X})-\sharp S(Z)=\sharp S(X) \neq 0,
$$

we know that $\sharp S(Z) \neq 0$ or $\sharp S(\bar{X}) \neq 0$. If $\sharp S(Z) \neq 0$ then $Z$, and thus $\bar{X}$, has a $K$-rational point by the induction hypothesis, since $Z$ has strictly smaller dimension than $X$. If $\sharp S(\bar{X}) \neq 0$, then $\bar{X}$ also has a $K$-rational point because $\bar{X}$ is smooth and proper over $K$, so that $\bar{X}$ is of type (N). Thus $\bar{X}(K)$ is Zariski-dense in $\bar{X}$, because $K$ is a henselian valued field and therefore a large field by [30, 3.1] (applied to the set of localities $\mathcal{L}=\{K\}$ ). It follows that $X(K)$ is non-empty.

Remark 3.17. Large fields are also called ample or fertile in the literature. Every henselian valued field is large. In $[30,3.1]$, this fact is deduced from the implicit function theorem for henselian valued fields.

\section{G-MODELS}

Let $G$ be a finite group.

Definition 4.1. Let $X$ be a $K$-variety endowed with a good action of $G$. A Gmodel for $X$ is a flat $R$-variety $\mathcal{X}$ that carries a good action of $G$, endowed with a G-equivariant isomorphism

$$
\mathcal{X}_{K} \rightarrow X
$$

The following lemma was kindly suggested to us by the referee.

Lemma 4.2. Let $\mathcal{Z}$ be an $R$-variety. Assume either that $R$ is excellent, or that the generic fiber $\mathcal{Z}_{K}$ is geometrically reduced. Then the normalization morphism $\widetilde{\mathcal{Z}} \rightarrow \mathcal{Z}$ is finite. 
Proof. If $R$ is excellent, this follows from [23, 33.H], so it is enough to consider the case where $\mathcal{Z}_{K}$ is geometrically reduced. We denote by $\widehat{R}$ and $\widehat{K}$ the completions of $R$ and $K$, respectively. We may assume that $\mathcal{Z}$ is affine, integral and $R$-flat, and we set $A=\mathcal{O}(\mathcal{Z})$. We set $B=A \otimes_{R} \widehat{R}$. Then $B$ is $\widehat{R}$-flat and thus a subring of $A \otimes_{R} \widehat{K}$. Since $\mathcal{Z}_{K}$ is geometrically reduced, $B$ is reduced.

If $C$ is an $A$-algebra such that every element of $C$ is integral over $A$, then $C$ is finite over $A$ if and only if it is finitely generated. Thus if the normalization $\widetilde{A}$ of $A$ were not finite over $A$, then by adjoining elements from $\widetilde{A}$ to $A$, we could construct a strictly ascending chain

$$
A \varsubsetneqq A_{1} \varsubsetneqq A_{2} \varsubsetneqq \cdots
$$

of finite sub- $A$-algebras of the fraction field $\operatorname{Frac}(A)$ of $A$. Tensoring with the faithfully flat $R$-algebra $\widehat{R}$, we would obtain a strictly ascending chain

$$
B=A \otimes_{R} \widehat{R} \varsubsetneqq A_{1} \otimes_{R} \widehat{R} \varsubsetneqq A_{2} \otimes_{R} \widehat{R} \varsubsetneqq \cdots
$$

of finite sub- $B$-algebras of $\operatorname{Frac}(A) \otimes_{K} \widehat{K}$. The $\operatorname{ring} \operatorname{Frac}(A) \otimes_{K} \widehat{K}$ is a subring of the total ring of fractions of $B$. It follows that the elements of the chain (4.1) are all contained in the normalization of $B$, which is finite over $B$ by excellence of $\widehat{R}$. This contradicts the fact that (4.1) is strictly ascending. Thus $\widetilde{A}$ is finite over $A$.

Proposition 4.3. Let $X$ be a normal proper $K$-variety, endowed with a good action of $G$. Assume either that $R$ is excellent, or that $X$ is geometrically reduced. Then $X$ admits a proper $G$-model $\mathcal{X}$ over $R$.

Proof. Since the $G$-action on $X$ is good, the quotient $X / G$ is representable by a $K$-variety, and the projection morphism

$$
X \rightarrow X / G
$$

is finite and surjective [11, V.1.5 and V.1.8]. Since $X$ is proper over $K$, we know that $X / G$ is proper over $K[8,5.4 .3]$. Since taking $G$-quotients commutes with extension of the base field $K[11, \mathrm{~V} .1 .9]$, we know that $X / G$ is geometrically reduced if $X$ is geometrically reduced.

By Nagata's embedding theorem, $X / G$ admits a flat and proper model $\mathcal{Y}$ over $R$. Let $\mathcal{X}$ be the normalization of $\mathcal{Y}$ in $X$. Then $\mathcal{X}$ is $R$-flat, and $\mathcal{X}_{K}$ is canonically isomorphic to $X$. The normalization map $\mathcal{X} \rightarrow \mathcal{Y}$ is finite, by Lemma 4.2. 
Thus $\mathcal{X}$ is a flat proper model for $X$ over $R$. The action of $G$ on $X$ induces an action on the total field of functions $K(X)$ of $X$, and, since $G$ acts trivially on $\mathcal{Y}$, the $G$-action on $X$ extends uniquely to an action on $\mathcal{X}$. The $G$-action on $\mathcal{X}$ is good since $\mathcal{X}$ is finite over $\mathcal{Y}$ and $G$ acts trivially on $\mathcal{Y}$.

Definition 4.4. Let $X$ be a smooth $K$-variety, endowed with a good $G$-action. $A$ weak Néron $G$-model of $X$ is an $R$-smooth $G$-model $\mathcal{X}$ for $X$ such that the natural map

$$
\mathcal{X}\left(R^{s h}\right) \rightarrow X\left(K^{s h}\right)
$$

is bijective.

Proposition 4.5. If $X$ is a smooth and proper $K$-variety, endowed with a good $G$-action, then $X$ admits a weak Néron $G$-model.

Proof. By Proposition 4.3, $X$ admits a proper $G$-model $\mathcal{X}$ over $R$. The smoothening algorithm in the proof of $[3,3.4 .2]$ produces a canonical sequence of blow-ups

$$
\mathcal{X}^{(r)} \rightarrow \ldots \mathcal{X}^{(1)} \rightarrow \mathcal{X}^{(0)}=\mathcal{X}
$$

such that, for every $i$ in $\{0, \ldots, r-1\}$, the center $C_{i}$ of the blow-up

$$
\mathcal{X}^{(i+1)} \rightarrow \mathcal{X}^{(i)}
$$

is a reduced closed subscheme of the special fiber $\mathcal{X}_{k}^{(i)}$ and such that $\operatorname{Sm}\left(\mathcal{X}^{(r)}\right)$ is a weak Néron model for $X$. Inspecting the proof of $[3,3.4 .2]$, we see that the center $C_{i}$ is stable under any $R$-automorphism of $\mathcal{X}^{(i)}$. It follows that all the models $\mathcal{X}^{(i)}$ of $X$ carry a unique extension of the $G$-action on $X$ and that all the blow-up morphisms are $G$-equivariant. Moreover, the $G$-action on $\operatorname{Sm}\left(\mathcal{X}^{(r)}\right)$ is good, since the $G$-action on $\mathcal{X}$ is good and the blow-up morphisms are projective. We conclude that $\operatorname{Sm}\left(\mathcal{X}^{(r)}\right)$ is a weak Néron $G$-model of $X$.

Proposition 4.6. Let $X$ be a smooth $K$-variety with good $G$-action, and let $\mathcal{X}$ be a weak Néron $G$-model for $X$. Assume that the order $|G|$ of $G$ is prime to $p$. Then the $G$-fixed locus $\mathcal{X}^{G}$ is a weak Néron model for $X^{G}$.

Proof. The scheme $\mathcal{X}^{G}$ is smooth over $R$, by Proposition 2.1. Moreover, we have

$$
\mathcal{X}^{G}\left(R^{s h}\right)=\left(\mathcal{X}\left(R^{s h}\right)\right)^{G}=\left(X\left(K^{s h}\right)\right)^{G}=X^{G}\left(K^{s h}\right) .
$$

This shows that $\mathcal{X}^{G}$ is a weak Néron model for $X^{G}$. 


\section{Serre's question}

In $[32, \S 1]$, Serre raises the following question.

Question 5.1. Let $F$ be a field, and let $G$ be a finite $\ell$-group, for some prime $\ell$ invertible in $F$. Suppose that $G$ acts on $\mathbb{A}_{F}^{n}$ for some integer $n>0$. Does this action always admit an $F$-rational fixed point?

Remark 5.2. As Serre points out, even the case $F=\mathbb{Q}, n=3$ and $|G|=2$ is open [32, p.2].

It is clear that the condition that $\ell$ is invertible in $F$ cannot be omitted. For instance, for every field $F$ of characteristic $\ell>1$, the additive group $\mathbb{G}_{a}\left(\mathbb{F}_{\ell}\right)$ acts on $\mathbb{A}_{F}^{1}$ by translation, and this action does not admit a fixed point.

We now discuss some cases where Serre's question can be answered. We start by recalling in Sections 5.1 and 5.2 some cases that are due to Serre. In Section 5.3, we show that Serre's question has a positive answer in dimension $\leq 2$, as implied by Serre in Remark 5.2. We also discuss the methods Serre uses, which immediately yield the real case (see Section 5.4). Finally, we treat in Section 5.5 the case where the ground field is a henselian discretely valued field of characteristic zero, with algebraically closed residue field of characteristic different from $\ell$. We show that, in that case as well, Serre's question has a positive answer.

For the remainder of Section 5, we maintain the assumption that $G$ is a finite $\ell$-group, with $\ell$ a prime invertible in the field $F$.

\subsection{The case where $F$ is finite.}

Proposition 5.3 (see [32], proof of Theorem 1.2.). Let $F$ be a finite field, and let $X$ be an F-variety with $G$-action. Then

$$
|X(F)| \equiv\left|X^{G}(F)\right| \bmod \ell .
$$

In particular, if $X=\mathbb{A}_{F}^{n}$ for some integer $n>0$, then $X^{G}(F)$ is non-empty.

Proof. For every orbit $O$ of the $G$-action on $X(F)$, the cardinality of $O$ is a power of $\ell$. Thus, either $O$ consists of a single fixed point, or the cardinality of $O$ is divisible by $\ell$. Since the orbits form a partition of $X(F)$, we find

$$
|X(F)| \equiv\left|X^{G}(F)\right| \bmod \ell .
$$


If $X=\mathbb{A}_{F}^{n}$ then $|X(F)|=|F|^{n}$, and this value is not divisible by $\ell$. It follows that $X^{G}(F)$ is non-empty.

5.2. The case where $F$ is separably closed. Serre gives several proofs for this case. In fact, he makes the stronger assumption that $F$ is algebraically closed, but this is not necessary: if $X$ is a smooth $F$-variety with $G$-action, then $X^{G}$ is smooth (Proposition 2.1). Hence, if $F$ is separably closed, then $X^{G}$ has an $F$-rational point as soon as it is non-empty.

The proof of Theorem 1.2 in [32] uses a spreading out argument to reduce to the case where $F$ is finite. Four other proofs make use of étale cohomology with $\ell$-adic coefficients $[32, \S 7]$. Let us briefly recall three of them, since these arguments will reappear in another context below.

5.2.1. Multiplicativity of the Euler characteristic in tame coverings. Serre proves the following result.

Proposition 5.4 (Serre [32], Section 7.2). Let $F$ be any field, and let $X$ be an $F$-variety with good $G$-action. Then one has the following congruence of $\ell$-adic Euler characteristics:

$$
\chi_{c}(X) \equiv \chi_{c}\left(X^{G}\right) \bmod \ell .
$$

In particular, if $\ell$ does not divide $\chi_{c}(X)$, then $X^{G}$ is non-empty.

Proof. Case 1: $|G|=\ell$. Then $G$ acts freely on the open subvariety $Y=X \backslash X^{G}$ of $X$, so that the projection $Y \rightarrow Y / G$ is a finite étale covering of degree $\ell$. Since $\ell$ is different from the characteristic of $F$, one has

$$
\chi_{c}(Y)=\ell \cdot \chi_{c}(Y / G)
$$

(see e.g. [14]). By additivity of the Euler characteristic, one has

$$
\chi_{c}(X)=\chi_{c}\left(X^{G}\right)+\ell \cdot \chi_{c}(Y / G)
$$

and the result follows.

Case 2: general case. We use an induction on the cardinality of $G$. Assume that $|G|>\ell$, and that we know the result for all finite $\ell$-groups $H$ with $|H|<|G|$. Since all finite $\ell$-groups are solvable, the group $G$ admits a non-trivial normal subgroup $H$. Since

$$
X^{G}=\left(X^{H}\right)^{G / H}
$$


one has

$$
\chi_{c}\left(X^{G}\right) \equiv \chi_{c}\left(X^{H}\right) \equiv \chi_{c}(X) \bmod \ell
$$

Corollary 5.5. Assume that $F$ is separably closed, and let $X$ be a smooth $F$ variety with good $G$-action. If $\ell$ does not divide $\chi_{c}(X)$, then $X^{G}(F)$ is non-empty. In particular, if $X=\mathbb{A}_{F}^{n}$ for some integer $n>0$, then $X^{G}(F)$ is non-empty.

5.2.2. The Grothendieck-Lefschetz-Verdier trace formula. Assume that $F$ is separably closed. Let $X$ be an irreducible $F$-variety of dimension $n$ with $G$ action, and assume that $H_{c}^{i}\left(X, \mathbb{Q}_{\ell}\right)=0$ for $i \neq 2 n$. This condition is fulfilled, for instance, if $X$ is $\mathbb{A}_{F}^{n}$. Since $H_{c}^{2 n}\left(X, \mathbb{Q}_{\ell}\right)$ has dimension one, and $G$ acts trivially on this cohomology space, we find that $t(g)=1$ for every element $g$ of $G$, where

$$
t(g)=\sum_{i \geq 0}(-1)^{i} \operatorname{Trace}\left(g \mid H_{c}^{i}\left(X, \mathbb{Q}_{\ell}\right)\right) .
$$

On the other hand, the Grothendieck-Lefschetz-Verdier trace formula [12, III] implies that $t(g)=0$ if $g$ does not have a fixed point on $X$. It follows that the $G$-action on $X$ admits a fixed point if $G$ is cyclic. If $X$ is smooth, there exists even an $F$-rational fixed point, by smoothness of $X^{G}$.

5.2.3. Smith theory. This approach yields the most detailed information about the fixed locus of $G$. In $[32,7.5]$, Serre proves the following result, using Smith theory (again, Serre makes the stronger assumption that $F$ is algebraically closed, but the case where $F$ is separably closed follows from this by invariance of étale cohomology under purely inseparable base change).

Theorem 5.6. Assume that $F$ is separably closed. Let $X$ be an F-variety with good $G$-action. Assume that $H^{i}(X, \mathbb{Z} / \ell)=0$ for $i>0$ and that $X$ is connected and non-empty. Then $H^{i}\left(X^{G}, \mathbb{Z} / \ell\right)=0$ for $i>0$ and $X^{G}$ is connected and non-empty.

Corollary 5.7. Assume that $F$ is separably closed, and let $n$ be a non-zero natural number. For any action of $G$ on $X=\mathbb{A}_{F}^{n}$, one has $H^{i}\left(X^{G}, \mathbb{Z} / \ell\right)=0$ for all $i>0$, and $X^{G}$ is smooth, connected and non-empty. In particular, $X^{G}(F)$ is non-empty.

Now let us look at some other cases where we can obtain a positive answer to Question 5.1. 
5.3. The case where $n \leq 2$. If $F$ is any field, the automorphism group of $\mathbb{A}_{F}^{1}=\operatorname{Spec} F[x]$ is easy to describe: a straightforward computation shows that it is the group of affine transformations

$$
x \mapsto a x+b
$$

with $a \in F^{\times}$and $b \in F$. Such an affine transformation $g$ has finite order $N>1$, with $N$ not divisible by the characteristic of $F$, if and only if $a$ is a primitive $N$-th root of unity. Then $x=b \cdot(1-a)^{-1} \in F$ is a fixed point, it is the only one and it is rational.

Proposition 5.8. Let $F$ be any field. If $G$ acts non-trivially on $\mathbb{A}_{F}^{1}$, then there exists a unique fixed point, and it is F-rational.

Proof. We argue by induction on $|G|$. If $|G|=\ell$ then $G$ is generated by a single element $g$ which acts on $\mathbb{A}_{F}^{1}$ as an affine transformation, so, as we have seen, there is a unique fixed point, which is rational.

We assume that $|G|>\ell$, and that we know the result for all finite $\ell$-groups $H$ with $|H|<|G|$. As all finite $\ell$-groups are solvable, $G$ admits a non-trivial normal subgroup $H$. If $H$ acts trivially on $\mathbb{A}_{F}^{1}$, then we can apply the induction hypothesis to the $G / H$-action on $\mathbb{A}_{F}^{1}$ and the result follows. If $H$ acts nontrivially, then by the induction hypothesis $\left(\mathbb{A}_{F}^{1}\right)^{H}$ is isomorphic to $\operatorname{Spec} F$, and the $G / H$-action on $\left(\mathbb{A}_{F}^{1}\right)^{H}$ is trivial.

Remark 5.9. We can also immediately deduce Proposition 5.8 from Corollary 5.7, since that corollary implies that the fixed locus $\left(\mathbb{A}_{F}^{1}\right)^{G}$ is smooth and geometrically connected over $F$.

Now we consider the case where $n=2$.

Lemma 5.10. Let $X, W$ be two normal irreducible varieties of dimension 2 over a field $F$, and let $Y \supset X, Z \supset W$ be normal compactifications. Let $g: X \rightarrow W$ be an isomorphism. If the irreducible components of $Z \backslash W$ of dimension one (with their reduced induced structure) are geometrically integral over $F$, then every point of indeterminacy $y$ of $g$ on $Y$ is F-rational. Moreover, the rational map $g^{-1}: Z \rightarrow Y$ contracts a one-dimensional irreducible component of $Z \backslash W$ to $y$.

Proof. Let $\Gamma_{g} \subset Y \times_{F} Z$ be the closure of the graph of $g$, with its reduced induced structure. Let $p$, resp. $q$ be the projection of $\Gamma_{g}$ to $Y$, resp. $Z$. Let $y$ be a closed 
point of $Y$, with residue field $\kappa(y)$. If $p^{-1}(y)$ has dimension zero, then by semicontinuity of fiber dimension $[10,13.1 .4]$, there exists an open neighbourhood $U$ of $y$ in $Y$ such that

$$
\left.p\right|_{p^{-1}(U)}: p^{-1}(U) \rightarrow U
$$

is quasi-finite. Since $Y$ is normal and

$$
\left.p\right|_{p^{-1}(X)}: p^{-1}(X) \rightarrow X
$$

is an isomorphism, we can deduce from $[9,4.4 .9]$ that $\left.p\right|_{p^{-1}(U)}$ is an isomorphism, so that $g$ is defined at $y$.

Hence, if $y$ is a point of indeterminacy of $g$, then $p^{-1}(y)$ contains an irreducible component of dimension one. Its image under $q$ is an irreducible closed subset $C$ of $Z$ of dimension one. This subset $C$ is disjoint from $W$, because $q^{-1}(W)$ is contained in $p^{-1}(X)$ (the graph of the isomorphism $g: X \rightarrow W$ is finite over $W$, and thus closed in $\left.Y \times{ }_{F} W\right)$. It follows that $C$ is an irreducible component of $Z \backslash W$. By our hypotheses, if we endow $C$ with its reduced induced structure, then $C$ is geometrically integral over $F$.

Since $Z$ is normal, the rational map $g^{-1}$ is defined on a dense open subscheme $C^{0}$ of $C$. By construction, $g^{-1}$ contracts $C^{0}$ to the point $y$. Since $C^{0}$ is geometrically integral over $F$, the existence of a morphism $C^{0} \rightarrow \operatorname{Spec} \kappa(y)$ implies that $\kappa(y)=F$ and $y$ is $F$-rational.

Lemma 5.11. Let $F$ be any field, and consider an action of $G$ on $X=\mathbb{A}_{F}^{2}$. Assume that the fixed locus $X^{G}$ has dimension one. Then the following properties hold.

(1) If $D$ is the regular completion of $X^{G}$, then $D \backslash X^{G}$ consists of a unique point $x_{\infty}$, which is rational or purely inseparable over $F$. Moreover, if $F^{a}$ is an algebraic closure of $F$, then the normalization $D^{\prime}$ of $D \times{ }_{F} F^{a}$ is isomorphic to $\mathbb{P}_{F^{a}}^{1}$.

(2) Assume that $G$ is cyclic, and let $g$ be a generator. Let $Y$ be a smooth compactification of $X$ such that the irreducible components of $Y \backslash X$ are smooth curves that intersect each other only at F-rational points. Denote by $C$ the closure of $X^{G}$ in $Y$, and assume that the birational map

$$
g: Y \rightarrow Y
$$


is defined at the unique point $c_{\infty}$ of $C \backslash X^{G}$. Then $X^{G}$ is isomorphic to $\mathbb{A}_{F}^{1}$.

Proof. (1) We denote by $F^{a}$ an algebraic closure of $F$. By Corollary 5.7, we know that $X^{G}$ is smooth and geometrically connected, and that

$$
H^{1}\left(X^{G} \times_{F} F^{a}, \mathbb{Z} / \ell\right)=0 .
$$

Hence, $X^{G}$ is a smooth geometrically connected affine curve and $\chi_{c}\left(X^{G}\right)=1$.

The normalization morphism $n: D^{\prime} \rightarrow D \times{ }_{F} F^{a}$ is an isomorphism over the smooth open subscheme $X^{G} \times{ }_{F} F^{a}$ of $D \times_{F} F^{a}$. We have

$$
1<\chi_{c}\left(X^{G}\right)+\chi_{c}\left(D^{\prime} \backslash n^{-1}\left(X^{G} \times_{F} F^{a}\right)\right)=\chi_{c}\left(D^{\prime}\right) \leq 2 .
$$

It follows that $D^{\prime}$ is isomorphic to $\mathbb{P}_{F}^{1}$ and that $D^{\prime} \backslash n^{-1}\left(X^{G} \times_{F} F^{a}\right)$ consists of a unique point. This implies that $D \backslash X^{G}$ consists of a unique closed point $x_{\infty}$, which is rational or purely inseparable over $F$.

(2) By (1), it is enough to show that $C$ is smooth over $F$ and that $c_{\infty}$ is separable over $F$ : then $c_{\infty}$ is $F$-rational and $C \cong D$ is isomorphic to $\mathbb{P}_{F}^{1}$.

For every $h \in G$, we denote by $U_{h}$ the domain of definition of the birational map

$$
h: Y \rightarrow Y
$$

and we set

$$
U=\bigcap_{h \in G} U_{h}, \quad V=\bigcap_{h \in G}\{x \in U \mid h(x) \in U\} .
$$

Then $V$ is an open subset of $Y$ that contains $X \cup\left\{c_{\infty}\right\}$, and the $G$-action on $X$ extends uniquely to a $G$-action on $V$, which stabilizes $V \backslash X$. By definition, the fixed locus $V^{G}$ contains $C$. By Proposition 2.1, $V^{G}$ is smooth over $F$. This implies that $C$ is a connected component of $V^{G}$, which is smooth over $F$.

If $c_{\infty}$ lies on more than one irreducible component of $Y \backslash X$, then by assumption, $c_{\infty}$ is $F$-rational. If $c_{\infty}$ lies on precisely one irreducible component $E$ of $Y \backslash X$, then $G$ acts on $V \cap E$, and $c_{\infty}$ is an isolated fixed point of this action. Applying Proposition 2.1 to the $G$-action on $V \cap E$, we see that $c_{\infty}$ is smooth, thus separable over $F$.

Theorem 5.12. Let $F$ be any field. For any $G$-action on $X=\mathbb{A}_{F}^{2}$, the fixed locus $X^{G}$ is isomorphic to $\mathbb{A}_{F}^{m}$, with $m \in\{0,1,2\}$. In particular, $X^{G}$ has an $F$-rational point. 
Proof. By Proposition 5.8 and an induction argument as in the proof of Proposition 5.8, we may assume that $|G|=\ell$. We may also assume that $X^{G}$ has dimension one, since the case of dimension two is obvious, and the case of dimension zero follows from Theorem 5.6. We now prove the theorem under these assumptions.

Let $g$ be a generator of $G$. It defines an automorphism of $X=\mathbb{A}_{F}^{2}$ and, thus, a birational map

$$
g: \mathbb{P}_{F}^{2} \rightarrow \mathbb{P}_{F}^{2}
$$

Denote by $C_{0}$ the closure of $X^{G}$ in $\mathbb{P}_{F}^{2}$. By Lemma 5.11 , the boundary $C_{0} \backslash X^{G}$ consists of a unique point $c_{0}$.

If $g$ is defined at $c_{0}$, then Lemma 5.11 implies that $X^{G}=\mathbb{A}_{F}^{1}$. Hence, we may assume that $g$ is not defined at $c_{0}$. Then $c_{0}$ is $F$-rational by Lemma 5.10, applied to $W=X=\mathbb{A}_{F}^{2}$ and $Y=Z=\mathbb{P}_{F}^{2}$.

Let $h_{1}: Y_{1} \rightarrow \mathbb{P}_{F}^{2}$ be the blow-up at $c_{0}$, and consider the birational map

$$
g \circ h_{1}: Y_{1} \rightarrow \mathbb{P}_{F}^{2} \text {. }
$$

We denote by $C_{1}$ the closure of $X^{G}$ in $Y_{1}$, and by $c_{1}$ the unique point of $C_{1} \backslash X^{G}$. If $g \circ h_{1}$ is not defined at $c_{1}$, then $c_{1}$ is $F$-rational by Lemma 5.10 , and we consider the blow-up

$$
h_{2}: Y_{2} \rightarrow Y_{1}
$$

at $c_{1}$.

If we continue in this way, then by resolution of indeterminacies [21, 9.2.7], we will eventually construct a sequence $\left(Y_{i}, C_{i}, c_{i}\right)$ with $i=0, \ldots, N$ and morphisms $h_{i}: Y_{i} \rightarrow Y_{i-1}$ for $i=1, \ldots, N$ that satisfy the following properties:

- $Y_{0}=\mathbb{P}_{F}^{2}, Y_{i}$ is a smooth compactification of $\mathbb{A}_{F}^{2}$ for every $i$, and $Y_{i} \backslash \mathbb{A}_{F}^{2}$ is a chain of $(i+1)$ copies of $\mathbb{P}_{F}^{1}$ that intersect at $F$-rational points,

- $C_{i}$ is the closure of $X^{G}$ in $Y_{i}$ and $c_{i}$ is the unique point of $C_{i} \backslash X^{G}$,

- the point $c_{i}$ is $F$-rational for every $i<N$,

- $h_{i}: Y_{i} \rightarrow Y_{i-1}$ is the blow-up at $c_{i-1}$, for every $i \geq 1$,

- if we put $h=h_{1} \circ h_{2} \circ \ldots \circ h_{N}$, then the birational map

$$
g \circ h: Y_{N} \rightarrow \mathbb{P}_{F}^{2}
$$

is defined at $c_{N}$. We denote by $U$ the domain of definition of $g \circ h$. 
For every $i$ in $\{0, \ldots, N\}$, the action of $g$ on $\mathbb{A}_{F}^{2}$ defines a birational map

$$
g_{i}: Y_{i} \rightarrow Y_{i}
$$

We claim that, for some $i$, either $g_{i}$ or $g_{i}^{-1}$ will be defined at $c_{i}$. This suffices to prove the theorem, by Lemma 5.11.

So let us prove the claim. Assume that $g_{0}^{-1}=g^{-1}$ is not defined at $c_{0}$. Then, a fortiori, the rational map

$$
(g \circ h)^{-1}: \mathbb{P}_{F}^{2}-\rightarrow Y_{N}
$$

is not defined at $c_{0}$. By Lemma 5.10, this means that $g \circ h$ contracts an irreducible component of $Y_{N} \backslash \mathbb{A}_{F}^{2}$ to $c_{0}$. By [21, 9.2.1], the morphism $g \circ h: U \rightarrow \mathbb{P}_{F}^{2}$ factors through the blow-up $h_{1}: Y_{1} \rightarrow \mathbb{P}_{F}^{2}$ at $c_{0}$ (to be precise, in [21, 9.2.1] it is assumed that $U$ is proper over $F$, but this is not necessary: one can apply [21, 9.2.1] to a compactification of the morphism $g \circ h: U \rightarrow \mathbb{P}_{F}^{2}$ with regular domain).

Proceeding inductively, we see that either $g_{i}^{-1}$ is defined at $c_{i}$ for some $i>0$, or $g \circ h$ factors through a morphism $g_{N}: U \rightarrow Y_{N}$ so that $g_{N}$ is defined at $c_{N}$.

Remark 5.13. One can push the proof a bit further: if $g_{i}^{-1}$ is defined at $c_{i}$ for some $i$, then the proof of Lemma 5.11 shows that the whole $G$-action is defined on an open neighbourhood of $C_{i}$ in $Y_{i}$. Since blowing up $G$-fixed closed points is $G$-equivariant, we see that in all cases, the $G$-action is defined on an open neighbourhood of $C_{N}$ in $Y_{N}$.

Corollary 5.14. Let $F$ be any field, and let $H$ be a finite solvable group whose order is not divisible by the characteristic of $F$. For any $H$-action on $X=\mathbb{A}_{F}^{2}$, the fixed locus $X^{H}$ is isomorphic to $\mathbb{A}_{F}^{m}$, with $m \in\{0,1,2\}$. In particular, $X^{H}$ has an F-rational point.

Proof. This follows immediately from Proposition 5.8, Theorem 5.12 and the fact that $H$ has a composition series whose composition factors are cyclic groups with order a prime number different from the characteristic of $F$.

5.4. The case $F=\mathbb{R}$. Here we can use an argument similar to Section 5.2.1 to prove the following result.

Proposition 5.15. Let $X$ be an $\mathbb{R}$-variety with good $G$-action. We consider $X(\mathbb{R})$ with its real topology. Then one has the following congruence of singular 
Euler characteristics with compact supports:

$$
\chi_{c, \text { sing }}(X(\mathbb{R})) \equiv \chi_{c, \operatorname{sing}}\left(X(\mathbb{R})^{G}\right) \quad \bmod \ell .
$$

In particular, if $\ell$ does not divide $\chi_{c, \operatorname{sing}}(X(\mathbb{R}))$, then $X(\mathbb{R})^{G}$ is non-empty.

Proof. We can simply copy the proof of Proposition 5.4, using the fact that, if $|G|=\ell$ and $Y=X \backslash X^{G}$, the projection

$$
Y(\mathbb{R}) \rightarrow Y(\mathbb{R}) / G
$$

is a topological covering of degree $\ell$.

Corollary 5.16. Any $G$-action on $\mathbb{A}_{\mathbb{R}}^{n}$, with $n$ a non-zero natural number, admits an $\mathbb{R}$-rational fixed point.

5.5. The case where $F$ is a henselian discretely valued field, of residual characteristic $\neq \ell$. Recall that the motivic Serre invariant $S(\cdot)$ has been defined in Definitions 3.7 and 3.12 and that the rational volume $s(\cdot)$ has been defined in Definition 3.13 .

Theorem 5.17. Let $\ell$ be a prime number and $G$ a finite $\ell$-group. Let $K$ be a henselian discretely valued field whose residue field $k$ is perfect. Let $X$ be a $K$ variety with good $G$-action. Assume either that $K$ has characteristic zero, or that $X$ is smooth and proper over $K$.

(1) Assume that the characteristic of $k$ is different from $\ell$. Then one has

$$
s(X) \equiv s\left(X^{G}\right) \bmod \ell .
$$

In particular, if $\ell$ does not divide $s(X)$, then $X^{G}\left(K^{\text {sh }}\right)$ is non-empty.

(2) Assume that $k$ is finite, of cardinality $q$. Then one has

$$
\sharp S(X) \equiv \sharp S\left(X^{G}\right) \quad \bmod \operatorname{gcd}(\ell, q-1) .
$$

In particular, if the residue class of $\sharp S(X)$ in $\mathbb{Z} /(\ell, q-1)$ is non-zero (e.g. if $\ell$ divides $q-1$ and $\sharp S(X)=1)$, then $X^{G}(K)$ is non-empty.

Proof. First, assume that $K$ has characteristic zero. Then all the members of the congruences in (1) and (2) are additive with respect to equivariant closed immersions of $K$-varieties with $G$-action. More precisely, taking the $G$-fixed locus defines a ring morphism

$$
(\cdot)^{G}: K_{0}\left(G-\operatorname{Var}_{K}\right) \rightarrow K_{0}\left(\operatorname{Var}_{K}\right):[X] \mapsto\left[X^{G}\right] .
$$


Since the equivariant Grothendieck group $K_{0}\left(G-\operatorname{Var}_{K}\right)$ is generated by the classes of smooth and proper $K$-varieties [2, 7.1], it suffices to consider the case where $X$ is smooth and proper.

So suppose that $K$ has characteristic $\geq 0$, that $\ell$ is invertible in $k$, and that $X$ is smooth and proper over $K$. By Proposition 4.5, $X$ admits a weak Néron $G$-model $\mathcal{X}$ over $R$. By Proposition 4.6, we know that $\mathcal{X}^{G}$ is a weak Néron model for $X^{G}$. By definition, one has $s(X)=\chi_{c}\left(\mathcal{X}_{k}\right)$ and $s\left(X^{G}\right)=\chi_{c}\left(\mathcal{X}_{k}^{G}\right)$, so that (1) follows from Proposition 5.4.

Likewise, if $k$ is finite, of cardinality $q$, one has by definition $\sharp S(X)=|\mathcal{X}(k)|$ and $\sharp S\left(X^{G}\right)=\left|\mathcal{X}^{G}(k)\right|$ in $\mathbb{Z} /(q-1)$. On the other hand, by Proposition 5.3, one has $|\mathcal{X}(k)| \equiv\left|\mathcal{X}^{G}(k)\right| \bmod \ell$. Combined with Proposition 3.16, this shows (2).

Corollary 5.18. Let $K$ be a henselian discretely valued field of characteristic zero with residue field $k$. Let $\ell$ be a prime number, and let $G$ be a finite $\ell$-group. Consider an action of $G$ on $X=\mathbb{A}_{K}^{n}$, for some integer $n>0$.

(1) If $k$ is algebraically closed and of characteristic $\neq \ell$, then

$$
s\left(X^{G}\right) \equiv 1 \bmod \ell .
$$

In particular, the $G$-action on $\mathbb{A}_{K}^{n}$ admits a $K$-rational fixed point.

(2) If $k$ is finite, of cardinality $q$, and $\ell$ divides $(q-1)$, then

$$
\sharp S\left(X^{G}\right) \equiv 1 \bmod \ell .
$$

In particular, the $G$-action on $\mathbb{A}_{K}^{n}$ admits a $K$-rational fixed point.

Proof. This follows immediately from Example 3.15 and Theorem 5.17.

Question 5.19. Is it still true that $X^{G}(K) \neq \emptyset$ if we omit the condition that $\ell$ divides $(q-1)$ in Corollary 5.18(2)?

Even if $R$ has mixed characteristic, Theorem 5.17 is false if we do not assume that $\ell$ is invertible in $k$, as is shown by the following example.

Example 5.20. Let $k$ be an algebraic closure of $\mathbb{F}_{3}$, let $R=W(k), K=\operatorname{Frac}(R)$ and $G=\mathbb{Z} / 3$. Denote by $g$ the class of 1 in $G$. Consider the action of $G$ on

$$
X=\operatorname{Proj} K[x, y]
$$


defined by

$$
g(x: y)=(y:-x-y) .
$$

Let $K^{a}$ be an algebraic closure of $K$. The points of $X^{G}\left(K^{a}\right)$ are the points with homogeneous coordinates $(1: \omega)$ with $\omega^{2}+\omega+1=0$. Since $\mu_{3}(K)=\{1\}$ we find that $X^{G}(K)$ is empty. It follows that $S\left(X^{G}\right)=0$, while $S(X)=2$ in $K_{0}^{R}\left(\operatorname{Var}_{k}\right) /\left(\mathbb{L}_{k}-1\right)$.

Note that the $G$-action on $X$ extends uniquely to an action of $G$ on the weak Néron model

$$
\mathcal{X}=\operatorname{Proj} R[x, y]
$$

of $X$. The $G$-action on $\mathcal{X}_{k}$ has a fixed point (namely $(1: 1)$ ) but this fixed point does not lift to an element of $\mathcal{X}^{G}(R)=X^{G}(K)$. Such a situation cannot occur when $\ell$ is invertible in $k$, by smoothness of $\mathcal{X}^{G}$ over $R$.

\section{THE TRACE FORMUla}

Let $K, R$ and $k$ be as in Section 2 . If $k$ is algebraically closed and of characteristic zero, the rational volume $s(X)$ of a $K$-variety $X$ admits a cohomological interpretation in terms of a trace formula, which is similar to the Grothendieck-Lefschetz-Verdier trace formula that we've discussed in Section 5.2 .2 .

Theorem 6.1. Let $K$ be a strictly henselian discretely valued field with residue field $k$, and assume that $k$ has characteristic zero. Let $K^{a}$ be an algebraic closure of $K$, and choose a topological generator $\varphi$ of the absolute Galois group $G\left(K^{a} / K\right)$ of $K$. Then for every $K$-variety $X$, one has

$$
s(X)=\sum_{i \geq 0}(-1)^{i} \operatorname{Trace}\left(\varphi \mid H_{c}^{i}\left(X \times_{K} K^{a}, \mathbb{Q}_{\ell}\right)\right)
$$

for every prime $\ell$.

Proof. If $K$ is complete, this is $[24,6.5]$. The proof remains valid in the general case. Alternatively, one can observe that both terms of the trace formula are invariant under completion of $K$, by Lemma 3.10, invariance of $\ell-$ adic cohomology under algebraically closed field extensions, and the fact that $G\left(\widehat{K}^{a} / \widehat{K}\right) \rightarrow G\left(K^{a} / K\right)$ is an isomorphism. 
There are partial results in this direction in arbitrary characteristic; see [25, $\S 4]$.

Proposition 6.2. We keep the assumptions of Theorem 6.1. Let $\ell$ be a prime, and $G$ a finite $\ell$-group. If $X$ is a smooth geometrically connected $K$-variety with good $G$-action such that

$$
H^{i}\left(X \times{ }_{K} K^{a}, \mathbb{Z} / \ell\right)=0
$$

for all $i>0$, then

$$
s(X)=s\left(X^{G}\right)=1
$$

In particular, $X^{G}$ has a $K$-rational point.

Proof. By Theorem 5.6, we know that

$$
H^{i}\left(X^{G} \times_{K} K^{a}, \mathbb{Z} / \ell\right)=0
$$

for all $i>0$, and that $X^{G}$ is geometrically connected. It follows that

$$
H^{i}\left(X^{G} \times_{K} K^{a}, \mathbb{Q}_{\ell}\right)=0
$$

for all $i>0$. The fixed locus $X^{G}$ is smooth over $K$ by Proposition 2.1, so that we can apply Poincaré duality, and we obtain that

$$
H_{c}^{i}\left(X^{G} \times_{K} K^{a}, \mathbb{Q}_{\ell}\right)=0
$$

for all $i \neq 2 d$, where $d$ denotes the dimension of $X^{G}$. Since $G\left(K^{a} / K\right)$ acts trivially on

$$
H_{c}^{2 d}\left(X^{G} \times_{K} K^{a}, \mathbb{Q}_{\ell}\right) \cong \mathbb{Q}_{\ell}(-d),
$$

Theorem 6.1 implies that $s\left(X^{G}\right)=1$. The same argument, where one assumes the $G$-action on $X$ to be trivial, shows that $s(X)=1$.

Corollary 6.3. We keep the assumptions of Theorem 6.1. Let $n$ be a non-zero natural number. Let $\ell$ be a prime, and $G$ a finite $\ell$-group. For any action of $G$ on $X=\mathbb{A}_{K}^{n}$, one has

$$
s\left(X^{G}\right)=1 .
$$

Question 6.4. Does this Corollary also hold if $K$ has mixed characteristic and $\ell$ is invertible in $k$ ? (Recall that $s\left(X^{G}\right)$ is congruent to 1 modulo $\ell$ by Corollary 5.18.) Is it true that one has the stronger equality

$$
S\left(X^{G}\right)=1
$$

in $K_{0}\left(\operatorname{Var}_{k}\right) /\left(\mathbb{L}_{k}-1\right)$ if $k$ has characteristic zero? 


\section{Relation With CONJECtURES in AFFine GeOMEtry}

It is clear that Serre's question (5.1) is closely related to the study of the structure of the automorphism group of affine spaces. If the base field $F$ is the field $\mathbb{C}$ of complex numbers, this study is an important part of complex affine geometry. The following question is mentioned in [18].

Question 7.1 (Linearization Problem). Let $n$ be a non-zero natural number, and let $g$ be an automorphism of $\mathbb{A}_{\mathbb{C}}^{n}$ of finite order. Can we always find a coordinate system on $\mathbb{A}_{\mathbb{C}}^{n}$ such that $g$ becomes linear? In other words, is $g$ conjugate to an element of $G L_{n}(\mathbb{C})$ in the automorphism group $\operatorname{Aut}_{n, \mathbb{C}}$ of $\mathbb{A}_{\mathbb{C}}^{n}$ ?

The question has been answered affirmatively if $n<3$, but it is open for $n \geq 3$. In the cases $n=1$ and $n=2$, the automorphism group Aut ${ }_{n, \mathbb{C}}$ is well-understood, but its structure is much more complicated when $n \geq 3$. We've seen in Section 5.3 that, for every field $F$, the automorphisms of $\mathbb{A}_{F}^{1}$ are precisely the invertible affine transformations. In dimension $n=2$, the theorem of Jung-van der Kulk [19] states that the automorphism group $\mathrm{Aut}_{2, F}$ of $\mathbb{A}_{F}^{2}$ is the amalgamated product of the subgroup Aff $\mathrm{Af}_{2, F}$ of invertible affine transformations and the subgroup $\mathscr{J}_{2, F}$ of so-called triangular automorphisms over their intersection $\mathscr{B}_{2, F}=\operatorname{Aff}_{2, F} \cap \mathscr{J}_{2, F}$. The group $\mathscr{J}_{2, F}$ is also called the Jonquière subgroup. For a geometric proof and some historical background, we refer to [20]. The theorem of Jung-van der Kulk implies by an elementary induction argument on the length of an element of the amalgamated product

$$
\operatorname{Aff}_{2, F} * \mathscr{B}_{2, F} \mathscr{J}_{2, F}
$$

that every automorphism $g$ of $\mathbb{A}_{F}^{2}$ of finite order $N$ is conjugate in $A_{u t}, F$ to an element of $\mathrm{Aff}_{2, F}$ or an element of $\mathscr{J}_{2, F}$ (see the discussion of Theorem 7 in [18]). If $N$ is not divisible by the characteristic of $F$, then $[15,2.1]$ implies that $g$ is conjugate to an element of $\mathrm{Aff}_{2, F}$. Since we know by Corollary 5.5 that the automorphism $g$ admits a fixed point over a separable closure of $F$, it follows that $g$ is linearizable, i.e., conjugate to an element of $G L_{2}(F)$. This yields an alternative proof of Theorem 5.12 .

In dimension 3, we get the following conditional result.

Proposition 7.2. Assume that the Linearization Problem has a positive solution for $n=3$. Let $F$ be a field of characteristic zero, and let $G$ be a finite solvable 
group. For any action of $G$ on $X=\mathbb{A}_{F}^{3}$, the fixed locus $X^{G}$ is isomorphic to $\mathbb{A}_{F}^{m}$ with $m \in\{0,1,2,3\}$. In particular, $X^{G}(F)$ is non-empty.

Proof. We may assume that $F$ is a subfield of $\mathbb{C}$, and that $G$ acts non-trivially on $X$. By Proposition 5.8 and Theorem 5.12 and an induction argument as in the proof of Proposition 5.8, we may suppose that $G$ is cyclic. Let $g$ be a generator. By our assumption in the statement of the proposition, the action of $g$ on $\mathbb{A}_{\mathbb{C}}^{3}$ can be linearized by a suitable choice of coordinates. This implies that $X^{G} \times_{F} \mathbb{C}$ is isomorphic to $\mathbb{A}_{\mathbb{C}}^{m}$, with $m \in\{0,1,2\}$. But $\mathbb{A}_{\mathbb{C}}^{m}$ has no non-trivial forms over $F$. For $m=0$ this is obvious. For $m=1$ it can be shown as in the beginning of the proof of Lemma 5.11(1): if $C^{0}$ is a form of $\mathbb{A}^{1}$ over $F$, and $C$ is a smooth compactification, then singular cohomology of $C$ over $\mathbb{C}$ implies that $C \backslash C^{0}$ is geometrically irreducible, thus is a rational point. For $m=2$ it is proven in [33] and [17].

Question 7.3. Let $G$ be a finite solvable group, and let $F$ be a field of characteristic zero. Is it true that, for every integer $n>0$ and every $G$-action on $X=\mathbb{A}_{F}^{n}$, the fixed locus $X^{G}$ is isomorphic to $\mathbb{A}_{F}^{m}$ for some $m \in\{0, \ldots, n\}$ ?

This would be compatible with the results in Sections 5 and 6, in particular Corollary 5.7. Note that, in order to show that Question 7.3 has a positive answer, it suffices to deal with the case where $G$ is a cyclic group of prime order, by induction on the order of $G$.

It seems that, in order to answer Question 5.1 in full generality, the tools of the previous sections do not suffice, and that one should exploit the specific geometric structure of the affine space.

\section{REFERENCES}

[1] A. Białynicki-Birula. Remarks on the action of an algebraic torus on $k^{n}$. Bull. Acad. Polon. Sci. Sr. Sci. Math. Astronom. Phys. 14:177-181, 1966.

[2] F. Bittner. The universal Euler characteristic for varieties of characteristic zero. Compos. Math. 140(4):1011-1032, 2004.

[3] S. Bosch, W. Lütkebohmert, and M. Raynaud. Néron models, volume 21 of Ergebnisse der Mathematik und ihrer Grenzgebiete. Springer-Verlag, 1990.

[4] W. Burnside. Theory of groups of finite order. Cambridge, Cambridge University Press, 1897. 
[5] Séminaire Henri Cartan, 13 no. 1, 1960-1961. Familles d'espaces complexes et fondements de la géométrie analytique.

[6] B. Conrad. Irreducible components of rigid spaces. Ann. Inst. Fourier, 49(2):473-541, 1999.

[7] B. Edixhoven. Néron models and tame ramification. Compos. Math., 81:291-306, 1992.

[8] A. Grothendieck and J. Dieudonné. Eléments de Géométrie Algébrique, II. Publ. Math. Inst. Hautes Étud. Sci., 8:5-222, 1961.

[9] A. Grothendieck and J. Dieudonné. Eléments de Géométrie Algébrique, III. Première partie. Publ. Math. Inst. Hautes Étud. Sci., 11:5-167, 1961.

[10] A. Grothendieck and J. Dieudonné. Eléments de Géométrie Algébrique, IV. Troisième partie. Publ. Math. Inst. Hautes Étud. Sci., 28:5-255, 1966.

[11] A. Grothendieck. Revêtements étales et groupe fondamental. Séminaire de Géométrie Algébrique du Bois-Marie 1960-1961 (SGA1). Volume 224 of Lecture Notes in Mathematics. Berlin; New York: Springer-Verlag, 1971.

[12] A. Grothendieck. Cohomologie $\ell$-adique et fonctions L. Séminaire de Géométrie Algébrique du Bois-Marie 1965-1966 (SGA5). Volume 589 of Lecture Notes in Mathematics. Berlin; New York: Springer-Verlag, 1977.

[13] J. Igusa. An introduction to the theory of local zeta functions. Studies in Advanced Mathematics. AMS, 2000.

[14] L. Illusie. Théorie de Brauer et caractéristique d'Euler-Poincaré (d'après P. Deligne). In The Euler-Poincaré characteristic, volume 82 of Astérisque, pages 161-172. 1981.

[15] N. Ivanenko. Some classes of linearizable polynomial maps. J. Pure Appl. Algebra 126(13):223-232, 1998.

[16] B. Iversen. A Fixed Point Formula for Action of Tori on Algebraic Varieties. Invent. Math. 16, 229-236, 1972.

[17] T. Kambayashi. On the absence of nontrivial separable forms of the affine plane. J. Algebra 35:449-456, 1975.

[18] H. Kraft. Challenging problems on affine $n$-space. Séminaire Bourbaki, Vol. 1994/95. Astérisque No. 237 (1996), Exp. No. 802, 5, 295-317.

[19] W. van der Kulk. On polynomial rings in two variables. Nieuw Arch. Wisk. 1:33-41, 1953.

[20] S. Lamy. Une preuve géométrique du théorème de Jung. Enseign. Math. 48(3-4):291-315, 2002.

[21] Q. Liu. Algebraic geometry and arithmetic curves. Volume 6 of Oxford Graduate Texts in Mathematics. Oxford, Oxford University Press, 2002.

[22] F. Loeser and J. Sebag. Motivic integration on smooth rigid varieties and invariants of degenerations. Duke Math. J., 119:315-344, 2003.

[23] H. Matsumura. Commutative algebra, 2nd edition. Volume 56 of Mathematics Lecture Note Series. Benjamin/Cummings Publishing Co., Inc., Reading, Mass., 1980.

[24] J. Nicaise. A trace formula for varieties over a discretely valued field. J. Reine Angew. Math., 650:193-238, 2011.

[25] J. Nicaise. Geometric criteria for tame ramification. preprint, arXiv:0910.3812v2.

[26] J. Nicaise and J. Sebag. Motivic Serre invariants and Weil restriction. J. Algebra, 319(4):1585-1610, 2008. 
[27] J. Nicaise and J. Sebag. The Grothendieck ring of varieties. In: R. Cluckers, J. Nicaise and J. Sebag (editors). Motivic integration and its interactions with model theory and nonarchimedean geometry. Volume 383 of London Mathematical Society Lecture Notes Series. Cambridge, Cambridge University Press, 2011.

[28] J. Nicaise and J. Sebag. A note on motivic integration in mixed characteristic. arXiv:0912.4887.

[29] J. Nicaise and J. Sebag. Motivic invariants of rigid varieties, and applications to complex singularities. In: R. Cluckers, J. Nicaise and J. Sebag (editors). Motivic integration and its interactions with model theory and non-archimedean geometry. Volume 383 of London Mathematical Society Lecture Notes Series. Cambridge, Cambridge University Press, 2011.

[30] F. Pop. Embedding problems over large fields. Ann. of Math. (2), 144(1):1-34, 1996.

[31] J.-P. Serre. Classification des variétés analytiques $p$-adiques compactes. Topology, 3:409412, 1965.

[32] J.-P. Serre. How to use finite fields for problems concerning infinite fields. In: Arithmetic, geometry, cryptography and coding theory, Contemp. Math., 487, Amer. Math. Soc., Providence, RI, 2009, pages 183-193.

[33] I. R. Shafarevich. On some infinite dimensional groups. Rend. Mat. e Appl. (5), 25: 208-212, 1966.

Hélène Esnault

Universität Duisburg-Essen, Mathematik,

45117 Essen, Germany

E-mail: esnault@uni-due.de

Johannes Nicaise

Katholieke Universiteit Leuven, Departement Wiskunde,

Celestijnenlaan 200B, B-3001 Leuven, Belgium

E-mail: Johannes.Nicaise@wis.kuleuven.be 